ECONOMÍA: Teoría y PrÁctica • Nueva Época, número 45, julio-diciembre 2016, pp. 207-234, http://www.izt.uam.mx/economiatyp/ojs

\title{
Evidencia de ciclo político presupuestal en el Estado de México*
}

\section{Evidence of Politic Budgetary Cycles in the Estado de Mexico}

\author{
Pablo Mejía Reyes, ${ }^{* *}$ Marlen R. Reyes Hernández*** \\ y Blanca I. Melquiades Ramírez****
}

\section{RESUMEN}

En este documento se determina la presencia de ciclos políticos presupuestales (CPP) asociados a las elecciones de gobernador del Estado de México; México, durante el periodo 1980-2011. Para ello, se analizan las características de los datos de gasto público en busca de evidencia preliminar. Posteriormente, se estiman modelos autorregresivos extendidos que incorporan variables (dicótomas) electorales y se controlan mediante los efectos del ciclo económico. Los resultados muestran la presencia de CPP en el gasto administrativo y la deuda pública, pero sólo evidencia episódica de conductas oportunistas en los rubros de gasto total (elección de 1993) y transferencias (elecciones de 1987 y 1999).

Palabras clave: gasto público, deuda pública, ciclo político presupuestal, México. Clasificación JEL: E62, H39.

\begin{abstract}
The aim of this paper is to determine the existence of political budget cycles associated to the elections of governors in the Estado de Mexico (Mexico) over the period 1980-2011. To address the subject, the characteristics of the government expenditure data are analyzed in a first moment looking for preliminary evidence. Afterwards, we estimate extended autoregressive models that incorporate electoral (dummy) variables and control for the effects of the business cycle. The results show evidence of political budget cycle in administrative expenditure and public debt, but only episodic evidence of opportunistic behavior in total expenditure (election of 1993) and transfers (elections of 1987 and 1999).
\end{abstract}

Keywords: public expenditure, public debt, political budget cycle, Mexico. JEL classification: E62, H39.

* Fecha de recepción: 16/12/2013. Fecha de aprobación: 18/04/2016. Los autores agradecen los comentarios de dos dictaminadores anónimos, los cuales han contribuido a mejorar este documento. Toda la responsabilidad de los errores y omisiones, no obstante, es completamente de los autores.

** Universidad Autónoma del Estado de México. Correo electrónico: pmejiare@uaemex.mx.

*** Universidad Autónoma del Estado de México. Correo electrónico: mar.rey2010@hotmail. com.

**** Universidad Autónoma del Estado de México. Correo electrónico: blancaivette.melquiades (a)gmail.com. 


\section{INTRODUCCIÓN}

Desde la perspectiva macroeconómica tradicional, se considera como política económica toda actuación del gobierno dirigida a influir en la trayectoria temporal de alguna variable de la economía. En general, se asume que las medidas de política económica están encaminadas a la consecución de objetivos específicos deseables, como la eficiencia productiva, la equidad distributiva y la estabilidad y sostenibilidad del crecimiento, así como a evitar o corregir aquellos desequilibrios que, si crecen o perduran, pueden hacer imposible alcanzar dichos fines. En esta perspectiva, se percibe al gobierno como un ente benévolo que sabe lo que desean y necesitan los gobernados e intenta conseguirlo en aras de la maximización del bienestar social (Argandoña, Gámez y Mochon, 1996).

Sin embargo, no es difícil suponer que los gobernantes pueden tener otros fines menos aceptables socialmente, como la conservación del poder (la reelección), el aumento de los ingresos propios, el prestigio y la puesta en práctica de una ideología específica, entre otros. La posibilidad, entonces, de que el manejo de los instrumentos de política económica pueda responder a factores políticos vinculados a las elecciones ha dado lugar al análisis de lo que se ha llamado ciclo político económico (CPE), por un lado, y ciclo político presupuestal (CPP), por otro (Assael y Larraín, 1994).

La hipótesis central en la teoría del CPE es que los gobernantes tratan de inducir resultados que les favorezcan ante los ojos de los votantes (aumento del empleo, la producción y el ingreso, o bien, mantenimiento artificial del nivel del tipo de cambio $)^{1}$ mediante la instrumentación de políticas expansivas antes y durante el periodo electoral, con lo cual pretenden mejorar sus oportunidades de reelección. Sin embargo, los excesos de tales medidas se manifiestan pronto bajo la forma de desequilibrios comerciales y fiscales, y de alzas en los precios, lo que obliga a los responsables de la política económica a hacer los ajustes restrictivos necesarios para evitar problemas mayores. Como consecuencia, se genera un ciclo - una expansión antes de y durante los comicios, seguida de una contracción en el periodo poselectoral- inducido por la manipulación de la política económica derivada del deseo de mantener el poder (Muñoz, 2006). Debido a su evidente origen, este patrón se ha denominado ciclo político económico. En general, se

\footnotetext{
${ }^{1}$ Un tipo de cambio estable puede fungir como ancla nominal en el control de la inflación, en tanto que un tipo de cambio sobrevaluado no sólo puede contribuir aún más a mantener bajos los precios, sino que también puede generar la sensación de que se dispone de una moneda doméstica más valiosa.
} 
considera que estos ciclos son, además, de naturaleza oportunista, lo cual se refiere a que tienen su origen en el intento de los gobernantes por mejorar sus oportunidades de reelección. En este contexto, el conflicto de intereses se desarrolla entre los gobernantes y los votantes. ${ }^{2}$

Sin embargo, aunque bien fundamentados, los modelos de CPE encontraron poco sustento empírico a nivel internacional. Las investigaciones que siguieron al trabajo pionero de Nordhaus (1975), desde la de McCallum (1978) hasta la de Alesina (1989), no encontraron respaldo para esta hipótesis. Al respecto, Weatherford (1987) mantiene que aunque el modelo de CPE propone una razón atractiva para que desde las instancias del Poder Ejecutivo se manipule la economía como una forma de ganar votos, el resultado ocurre raras veces. Ante esta situación, la atención se ha desplazado de los resultados (visibles en variables macroeconómicas) a las intenciones de los gobernantes (manifiestas en el uso de los instrumentos de política económica), con especial énfasis en la manipulación de la política fiscal con fines electorales.

De esta forma, el fenómeno de interés pasó al CPP, definido por Shi y Svensson $(2002$, p. 1) como "[...] la fluctuación periódica en las políticas fiscales del gobierno, inducidas por el calendario electoral [...]". Así, aun cuando no haya evidencia contundente sobre los efectos macroeconómicos de la manipulación de la política económica con fines electorales, sí puede haber indicios claros acerca de las intenciones de los gobernantes de manejar los instrumentos fiscales de acuerdo a sus intereses políticos.

Es fundamental subrayar que el problema central del manejo electoral de la política fiscal es que cuando se le hace responder a objetivos distintos a los de la estabilización macroeconómica, se le resta eficacia y se le asocia al desperdicio de recursos públicos, empleados para beneficiar a partidos o grupos políticos específicos (Fontaine, 1983).

En el caso de México, existe una percepción generalizada de que los encargados del Ejecutivo en los diferentes niveles de gobierno manipulan sistemáticamente la política económica (en especial la fiscal), con el fin de que sus partidos se mantengan en el poder. Por ejemplo, y aun cuando no se ha logrado alcanzar un consenso definitivo, existe evidencia de que las crisis financieras y

\footnotetext{
${ }^{2}$ En otros casos, las oscilaciones económicas se derivan de las diferencias ideológicas (en relación al manejo de la economía) entre los partidos que aspiran a gobernar, en el marco de sistemas con alternancia democrática, lo cual da lugar a los llamados ciclos político-económicos partidistas. En este caso, el conflicto de intereses se desarrolla entre los diferentes grupos políticos con puntos de vista divergentes sobre la correcta orientación de la política económica (Hibbs, 1977).
} 
las recesiones de los años setenta, ochenta y noventa fueron generadas en parte por el manejo oportunista de diferentes instrumentos de política económica (Heath, 2000; Gámez, 2012). ${ }^{3}$ En un nivel más específico, existen algunos estudios que han aportado evidencia de la existencia de CPP en México (Magaloni, 2000; González, 2002; Gámez, 2010) y otros que han abordado este fenómeno a nivel de los estados del país o grupos de ellos (Gámez e Ibarra-Yúnez, 2009; Ramírez y Erquizio 2012). No obstante, aunque la evidencia que presentan constituye una contribución valiosa a la literatura, sus resultados no permiten hacer inferencias sobre algún estado en particular.

Por ello, el objetivo de esta investigación es identificar (o descartar) la presencia de CPP en el Estado de México durante los comicios para gobernador celebrados entre 1980 y 2011. Como es sabido, esa entidad es la más poblada del país y la segunda más importante de la economía nacional. Además, es considerada por diversos estudiosos como un laboratorio de la política nacional, no sólo por el porcentaje de votantes que representa, ${ }^{4}$ sino también por el conveniente hecho de que la elección del gobernador mexiquense se realiza un año antes que la de presidente de la República (González, 2008). Más aún, el estado de México siempre ha sido gobernado por el Partido Revolucionario Institucional (PRI), lo que puede imprimir rasgos adicionales a la lógica del CPP: lo que motiva a los gobernantes no sólo es el deseo de mantener a su partido en el poder, ${ }^{5}$ sino la necesidad de legitimar ante el electorado su permanencia, lo que en términos prácticos puede derivar en conductas oportunistas, tal como sugiere la teoría.

Con tal fin, este documento se divide en tres secciones. En la primera, se presenta una visión panorámica de las teorías que explican el CPP y se bosqueja un modelo que formaliza este planteamiento. También se discute una selección de la literatura empírica existente, enfatizando la relacionada con el caso mexicano. En la sección II se expone en detalle la metodología empírica empleada para

\footnotetext{
${ }^{3}$ Posiblemente los episodios más evidentes sean los de finales de los sexenios de Luis Echeverría, José López Portillo y Carlos Salinas de Gortari, en 1976, 1982 y 1994, respectivamente. Véase Magaloni (2000), González (2002) y Reyes, Mejía y Riguzzi (2013), para un análisis del CPE de estos periodos, y Cárdenas (1996), Lustig (2002) y Moreno-Brid y Ros (2010) para un análisis general de las crisis económicas de esos periodos.

${ }^{4}$ En 1980, la población y la producción del Estado de México representaban $11.3 \%$ y $9.6 \%$ de los totales nacionales respectivos, en tanto que, en 2010, las cifras correspondientes alcanzaban 13.5 y 9.2\% (Germán, 2005; Instituto Nacional de Estadística, 2013). Por su parte, la proporción de votantes del total nacional, en 2010, fue de 13.8\% (Instituto Federal Electoral, 2013).

${ }^{5}$ Dado que en México no existe reelección a ningún nivel del Ejecutivo, el gobernante sólo puede aspirar a que el candidato postulado por su partido gane la elección respectiva.
} 
identificar los CPP, en tanto que la sección III describe la información estadística utilizada y presenta y discute los resultados obtenidos para el Estado de México. Finalmente, se anotan las principales conclusiones.

\section{CiClo POLÍTICO PRESUPUESTAL: TEORÍA Y EVIDENCIA EMPÍRICA}

\section{Elementos teóricos}

Algunas teorías consideran que la acción del gobierno debe estar encaminada a estabilizar la economía con la finalidad de mejorar el bienestar social. Sin embargo, desde mediados de los años setenta, algunas argumentaciones sostienen que el Estado interviene en la economía real persiguiendo intereses que no son los del bienestar, sino que se asocian con el deseo de mantener el poder. Por ello, se reconoce, por ejemplo, que el gobierno utiliza los instrumentos fiscales, especialmente el gasto público, con el objetivo de inducir resultados que lo hagan ver como un gestor exitoso de la macroeconomía. Por ello, se considera necesario agregar factores políticos a los determinantes de los ciclos económicos tradicionalmente aceptados.

Gran parte de la literatura sobre ciclos económicos y políticos tiene su origen en los modelos teóricos desarrollados a mediados de los setenta. La primera generación de modelos parte de una caracterización de la economía basada en dos elementos clave. En primer lugar, la estructura económica se resume en una curva de Phillips aumentada con expectativas de inflación, al tiempo que se considera que los votantes valoran positivamente un mayor nivel de empleo y negativamente una mayor tasa de inflación. En segundo lugar, se asumen expectativas adaptativas, lo que se interpreta como miopía (o irracionalidad) de los votantes, ya que cuando el político adopta medidas expansivas, el votante no recuerda o no toma en consideración las políticas recesivas o ineficientes del pasado, y que con certeza se llevarán adelante en el futuro. En esta primera generación nace también la división analítica de los CPE en oportunistas y partidistas. ${ }^{6}$

A mediados de los años ochenta surge una nueva generación de modelos de CPE, tanto oportunistas como partidistas, que incorporan expectativas raciona-

\footnotetext{
${ }^{6}$ El trabajo de Hibbs (1977) es el punto de partida para el estudio sistemático del ciclo ideológico o partidista. Su principal supuesto es que los partidos políticos se mueven exclusivamente por motivaciones ideológicas y maximizan diferentes funciones objetivo que le dan distinto peso al costo del desempleo en relación con la inflación. Los votantes actúan de acuerdo con sus preferencias ideológicas y sus intereses económicos.
} 
les. ${ }^{7}$ El supuesto es que las políticas económicas sistemáticas son completamente anticipadas por los votantes racionales de tal forma que, en equilibrio, éstos no pueden ser permanentemente engañados. Sin embargo, problemas de información asimétrica, que pueden afectar a los gobernantes y al electorado, permiten la aparición de CPE aun en el caso de que los votantes elaboren sus expectativas racionalmente.

La falta de evidencia contundente que probara la existencia de los CPE provocó un desplazamiento de la atención hacia el CPP, como se mencionó antes. En los modelos representativos de esta etapa (Rogoff y Sibert, 1988; Rogoff, 1990), el funcionario oportunista manipula la política de gasto en años electorales sólo con el objeto de mostrar que es competente y, de esta manera, aumentar su oportunidad de ganar las elecciones. Según estos modelos, bastaría con que el gobierno en cuestión cuente con poder para decidir sobre sus ingresos y sus gastos para incidir sobre los resultados electorales. En esencia, el argumento de partida es que el manejo de los instrumentos fiscales es más propicio para la aparición de conductas oportunistas por varias razones: el gobierno tiene un mayor grado de control, especialmente del gasto público, y la política fiscal tiene efectos reales, aun si es anticipada, y puede afectar las condiciones de grupos específicos de votantes, incluso si no tiene efectos agregados (Drazen, 2001). ${ }^{8}$ Así pues, la teoría del CPP sustenta que antes de las elecciones, los gobiernos (en todos los niveles) con frecuencia incrementan el gasto de consumo, los subsidios y el gasto en obras públicas, o bien, disminuyen los impuestos o difieren el gasto hacia partidas más visibles. ${ }^{9}$

En este marco teórico, la investigación de Tufte (1978) es reconocida como la primera en presentar evidencia de manipulación preelectoral de distintos instrumentos de política fiscal. De su análisis, así como de otros trabajos empíri-

\footnotetext{
${ }^{7}$ En los modelos partidistas o ideológicos con expectativas racionales, los partidos interactúan con los electores, quienes están al tanto de que los partidos tienen distintas funciones objetivo, y en los que lo importante serán las políticas inesperadas. En general, estos modelos se basan en el supuesto de rigideces nominales. Para mayores detalles, véase Alesina (1987) y Alesina y Sachs (1988).

${ }^{8}$ Además, la discrecionalidad en el manejo de la política monetaria disminuye cuando el banco central es autónomo.

${ }^{9}$ No existe consenso en la literatura sobre cuál rubro es el más visible: Rogoff (1990) sostiene que el gasto se sesga hacia el corriente, mientras que Schuknecht (2000) supone que en países en desarrollo se prioriza el gasto en bienes de capital, argumentando que el hecho de inaugurar grandes obras antes de las elecciones y detenerlas después de ellas parece más fácil que aumentar el gasto corriente, ya que este último puede traer aparejados compromisos de mediano y largo plazos.
} 
cos relevantes realizados posteriormente, (Rogoff y Sibert, 1988; Rogoff 1990; Schucknecht, 2000) se pueden identificar dos hechos bien reconocidos. En primer lugar, el ciclo se refleja en un comportamiento irregular de las variables presupuestales (gastos personales y de inversión, impuestos y deuda pública, entre otros), que se distribuye discrecionalmente en dos periodos: el preelectoral (el año de los comicios y el inmediato anterior) y el postelectoral (los dos años siguientes a su celebración). En segundo lugar, el aumento de los gastos más visibles para los ciudadanos, el recorte de las tasas impositivas o el uso estratégico del endeudamiento durante el periodo anterior a la votación responden a motivaciones oportunistas.

En particular, Rogoff y Sibert (1988) se concentran en el manejo de la política fiscal con fines electorales, de manera que la capacidad del gobernante es definida en este caso por su habilidad para proveer bienes públicos, dadas ciertas restricciones presupuestarias. Los autores señalan que el gobernante reduce los impuestos o incrementa los gastos antes de las elecciones a fin de mostrar su capacidad para beneficiar a los ciudadanos, aun cuando sólo sea temporalmente y eso genere déficit preelectorales.

Así, las decisiones de política económica tomadas por el gobernante en turno pueden no ser óptimas desde el punto de vista del bienestar social, aunque, desde su perspectiva, las distorsiones que aquéllas generen sean más que compensadas por dos resultados previsibles: los privilegios que disfrutará de permanecer en el poder y el mayor bienestar económico que con certeza será capaz de producir en el futuro periodo constitucional (por considerarse a sí mismo el más capaz). Los votantes racionales, pero imperfectamente informados, por su parte, reeligen al gobernante competente como reacción a las acciones que éste realiza para hacer patente su gran competencia, aun cuando están perfectamente conscientes de los costos para el bienestar social involucrados en el proceso de destacar su imagen. Una vez que los votantes identifican al gobernante como competente, lo reelegirán porque la utilidad esperada en el siguiente periodo constitucional es mayor con él en el poder (cuya competencia es alta, con certeza) que con el candidato opositor (quien tiene menor probabilidad de ser competente). No obstante, existe la posibilidad de que los votantes no tengan la información suficiente para distinguir entre el candidato competente y el incompetente, en cuyo caso los efectos del CPP sobre el bienestar serán claramente negativos. ${ }^{10}$

\footnotetext{
${ }^{10}$ Véase el trabajo de Lohmann (1998) para extensiones del modelo de Rogoff(1990), donde se considera esta posibilidad.
} 


\section{Evidencia empírica}

En general, la metodología empírica empleada en la literatura se basa en la estimación de modelos autorregresivos (AR), cuyo orden se determina utilizando los criterios de información de Akaike o de Schwarz o el enfoque de lo general a lo específico -como Ogura (2000)-; cuando la muestra consta de diferentes estados o países, lo común es emplear modelos para datos de panel. Desde una perspectiva teórica, la dinámica del CPP supone que el efecto de las elecciones -antes y durante ellas- sobre las variables fiscales es positivo, mientras que el impacto después de celebradas es negativo. En esa lógica, para evaluar la presencia del CPP, se incorporan variables dicótomas que tratan de captar diferencias en el crecimiento de las variables antes, durante y después de las elecciones, aunque cada trabajo considera un número diferente de periodos en torno a ellas.

Específicamente, Remmer (1991) analiza los efectos electorales sobre el balance fiscal en ocho países de América Latina y concluye que la dirección de tales efectos es heterogénea. Vínculos entre comicios y cambios presupuestales pueden establecerse sólo en el caso de Venezuela. Además, la variable electoral estadísticamente significativa sugiere un patrón opuesto al postulado por la teoría. Por su parte, Ogura (2000) sustenta, en el caso Brasil, que la evidencia hallada por él sobre el aumento de los gastos salariales del gobierno durante su primer año de gestión se justifica a partir de la idea de que aunque ese aumento puede ocurrir antes de las elecciones, su impacto se presenta al año siguiente. Larraín y Assael (1997), a su vez, analizan el caso de Chile y encuentran que en todas las elecciones estudiadas, excepto en la de diciembre de 1989, el déficit público real tuvo un comportamiento consistente con las predicciones del modelo del CPP.

En el caso específico de México, algunos trabajos han observado de forma común que el partido en el poder realiza gastos extraordinarios antes de la justa electoral. Por ejemplo, González (2002) estima modelos autorregresivos para el periodo 1957-1997 y encuentra evidencia de manipulación electoral sistemática de la inversión en infraestructura y un efecto positivo del grado de democracia sobre la magnitud del CPP. Sin embargo, no halla evidencia consistente en las transferencias, los ingresos y el gasto total. Por su parte, Magaloni (2000) utiliza un enfoque similar para el periodo 1983-1998 y reporta evidencia de CPP en diferentes indicadores de gasto. Sin embargo, dicha autora aclara que cuando el modelo se estima considerando el desempleo como variable de control, la caída en el gasto público pierde significación estadística, por lo que sostiene que existe la posibilidad de que el resultado sea explicado por el manejo fiscal acorde 
con el ciclo económico, en particular por el descenso del crecimiento durante el primer año del periodo presidencial.

Reyes y Mejía (2016) determinan la presencia de un CPP en torno a las elecciones presidenciales durante el periodo 1980-2014. Para ello, estimaron modelos autorregresivos y de promedios móviles, extendidos para tomar en cuenta los efectos de factores económicos, para el gasto público total, el gasto público programable y los componentes de éste. Entre los resultados más importantes destacan la evidencia de CPP en el gasto público total, en el gasto corriente $\mathrm{y}$, sobre todo, en el gasto de capital. A su vez, el gasto programable, y algunos rubros de éste, como inversión física, transferencias, bienes muebles e inmuebles, adquisiciones y otros, sólo proveen evidencia parcial de CPP, con ajustes posteriores a la elección.

Por otra parte, un par de estudios analizan la presencia de CPP en los estados mexicanos. ${ }^{11}$ En primer lugar, Gámez e Ibarra-Yúnez (2009) argumentan que, en general, existe evidencia de CPP durante el periodo 1997-2004 en los 31 estados (excluyendo la Ciudad de México), ${ }^{12}$ aunque con algunas diferencias: se detecta un incremento en el gasto en los años electorales, pero no en los previos, y un recorte en los años posteriores. Entre sus resultados, destaca el incremento de los subsidios antes y durante el año electoral y del rubro "Otros" durante los tres años del ciclo comicial. Sin embargo, no es posible detectar un comportamiento cíclico en los gastos en obras públicas alrededor de los años de elección, identificado en estudios anteriores como uno de los más propensos a ser manipulados electoralmente. De manera similar, Ramírez y Erquizio (2012) analizan el comportamiento del gasto público total, las transferencias, el gasto en obra pública y el gasto administrativo en 31 entidades federativas y 14 gobiernos estatales donde el PRI ha permanecido en el poder de manera ininterrumpida de 1993 a 2009. Sus resultados confirman aumentos del gasto total durante el año electoral en los 31 estados y, al igual que Gámez e Ibarra-Yúnez (2009), no encuentran evidencia de CPP en los gastos en obras públicas. Sin embargo, Ramírez y Erquizio (2012) estiman un aumento significativo en las transferencias cuando sólo se consideran los estados que han sido gobernados permanentemente por el PRI. ${ }^{13}$

\footnotetext{
${ }^{11}$ En ambos, se utilizan métodos de estimación para datos de panel.

${ }^{12}$ Estos autores miden la influencia del CPP en el nivel y la composición del gasto público estatal durante 47 procesos electorales.

${ }^{13}$ Asimismo, los autores reportan un incremento interanual de la deuda pública como factor explicativo de los aumentos de los gastos totales y de las transferencias en los 31 estados.
} 
En resumen, los estudios mencionados sugieren que existe evidencia del manejo electoral del gasto público y sus componentes, en diferentes países y estados de México, aunque la evidencia no es definitiva. En México, existe la percepción generalizada de que el gasto público se maneja con fines electorales, aun cuando la evidencia reportada en la literatura tampoco es contundente (Reyes y Mejía, 2016). En el Estado de México, con gobiernos permanentemente priistas, existe la misma sensación. No obstante, los estudios realizados han analizado la experiencia de los estados de la República Mexicana, o de los gobernados por el mismo PRI, en conjunto, lo que impide conocer la experiencia de casos específicos, la cual, evidentemente, puede ser muy diferente del resto y puede perderse en el promedio de estudios colectivos. En ello estriba la aportación del presente documento al abordar el análisis de este importante estado.

\section{MetodologíA}

En esta sección se presenta la metodología para desarrollar el análisis empírico del CPP en el Estado de México. En línea con los numerosos trabajos existentes en la literatura, se estiman modelos autorregresivos que se extienden para incorporar variables electorales y de control; las primeras tratan de captar los efectos de las elecciones y las segundas los efectos del ciclo económico sobre el comportamiento de diferentes rubros del gasto público. Dadas las dificultades para identificar la presencia del CPP en el comportamiento de diferentes rubros de gasto público de México, ${ }^{14}$ en este trabajo se utilizaron dos especificaciones alternativas para las variables electorales. En primer lugar, se emplea la especificación convencional, para el modelo 1 , expresada en los siguientes términos:

$$
g_{k t}=\phi_{0}+\sum_{i=1}^{3} \phi_{i} g_{k t-i}+\sum_{i=0}^{3} \alpha_{i} y_{t-i}^{n}+\sum_{i=0}^{3} \beta_{i} y_{t-i}^{m}+\gamma_{\alpha} E_{\tau-1}+\gamma_{e} E_{\tau}+\gamma_{d} E_{\tau+1}+\varepsilon_{t},
$$

donde las variables están expresadas en tasas de crecimiento anual y $g_{k}$ representa el $k$-ésimo componente del gasto público; $y^{n}$ y $y^{m}$ corresponden al producto interno bruto (PIB) nacional y estatal, y se utilizan para captar los efectos del ciclo económico nacional y estatal, respectivamente; $\tau$ denota la fecha de la elección,

\footnotetext{
${ }^{14}$ Reyes y Mejía (2016) argumentan que la discrecionalidad en el manejo del gasto hace que las series de sus componentes exhiban características estadísticas poco deseables (con distribuciones sesgadas y leptocúrticas, principalmente), que las hacen difíciles de modelar. La metodología econométrica usada aquí extiende la de estos autores.
} 
de modo que $E_{\tau-1}, E_{\tau}$ y $E_{\tau+1}$ corresponden a variables dicótomas que toman el valor de 1 un año antes, durante y un año después de las elecciones, respectivamente, y 0 en cualquier otro periodo. ${ }^{15}$ Los parámetros a estimar son $\phi_{i}, \alpha_{i}, \mathrm{y} \beta_{i}$, así como $\gamma_{a}, \gamma_{e}$ y $\gamma_{d}$. Se espera que los parámetros $\phi_{i}$ cumplan con las condiciones de estacionariedad del componente autorregresivo. A su vez, no existen restricciones sobre los coeficientes del producto nacional y estatal dado que puede haber una relación positiva o negativa con el gasto, dependiendo de si la política fiscal ha sido pro- o contracíclica. Ahora bien, para que haya evidencia de CPP, se requiere que $\gamma_{a}, \gamma_{e}>0$ y $\gamma_{d}<0$; en otras palabras, se requiere que la media de la tasa de crecimiento del gasto sea mayor en el año previo y en el de la elección y menor (en sentido estricto, negativa) en el año poselectoral. Por último, se asume que $\varepsilon_{t}$ sigue un proceso de ruido blanco gaussiano, es decir, $\varepsilon_{t} \sim$ iid $N\left(0, \sigma^{2}\right)$.

En segundo lugar, las variables dicótomas se definen de manera que puedan captar conductas oportunistas en cada una de las elecciones celebradas en el Estado de México. Para ello, las variables electorales $F_{i}$ toman los valores de 1, 1 $y-1$ en el año inmediato anterior a la $i$-ésima elección $(t=\tau-1)$, el año de las elecciones $(t=\tau)$ y el año posterior a ellas $(t=\tau+1)$, y 0 en cualquier otro periodo. La estimación en este caso (modelo 2) es la siguiente:

$$
g_{k t}=\phi_{0}+\sum_{i=1}^{3} \phi_{i} g_{k t-i}+\sum_{i=0}^{3} \alpha_{i} y_{t-i}^{n}+\sum_{i=0}^{3} \beta_{i} y_{t-i}^{m}+\sum_{i=1}^{4} \delta_{i} F_{i t}+\varepsilon_{t},
$$

donde se consideran las mismas variables de control que en la ecuación (1) y denota los coeficientes que miden el efecto de las cuatro distintas elecciones en la tasa media de crecimiento del gasto público. ${ }^{16}$ Dado que la aparición de CPP

${ }^{15}$ En estudios donde se analizan datos de panel, se incluyen otras variables de control para tomar en cuenta las diferencias en los niveles de desarrollo o en el marco institucional de los países o estados considerados, tales como el PIB per cápita, indicadores sociales y la competencia política (Gámez e Ibarra-Yúnez, 2009; Ramírez y Erquizio, 2012). En este estudio, donde sólo se analiza la experiencia de un estado, no se incluyen variables de este tipo porque esos aspectos suelen cambiar relativamente poco en el tiempo, además de que se carece de información estadística para algunos de ellos para el periodo analizado. No obstante, los efectos de los cambios institucionales pueden ser captados por la especificación del modelo 2 .

${ }^{16}$ Se incluyeron también dos variables electorales que pretendían medir los efectos parciales de las elecciones de 1981 y 2011, años que se encuentran al principio y final de la muestra. Sus coeficientes no fueron significativos en ningún caso, por lo que se excluyeron de las estimaciones finales. Además, los residuos de los modelos estimados mostraron valores muy grandes en varios casos, generando distribuciones leptocúrticas y, por tanto, no normales. Para eliminarlos, se incluyeron variables dicótomas adicionales, las cuales a veces pudieron relacionadas con el CPP, como se discute más adelante. 
puede variar en el tiempo, en función de los cambios en las instituciones electorales, o en el nivel de educación y participación política del electorado, se esperaría que los valores estimados de este parámetro fueran cada vez menores y que perdieran significación estadística.

Estas dos especificaciones se emplean para identificar las presencia de CPP en distintos componentes del gasto público mexiquense. La estrategia de estimación sigue el enfoque de lo general a lo específico: se inicia estimando el modelo general en una primera etapa y eliminando, en una segunda fase, la variable con el coeficiente menos significativo estadísticamente en una segunda fase. Se estima el nuevo modelo y se procede nuevamente de esta forma hasta que el modelo resultante contenga solamente coeficientes con, al menos, $10 \%$ de significación estadística (véase Charemza y Deadman, 1997).

\section{EVIDENCIA de CPP EN El Estado de MÉXICo}

En esta parte, se presentan las características generales de la información estadística utilizada y, a partir de ellas, se aporta la evidencia preliminar sobre la existencia de CPP en distintos componentes del gasto público del Estado de México. Posteriormente, se estiman los modelos formulados en la sección anterior y se contrastan los resultados con los de otros estudios.

\section{Información estadística y evidencia preliminar de CPP}

Para determinar si han existido CPP asociados a las elecciones de gobernador del Estado de México durante el periodo 1980-2011, se utiliza información sobre el gasto público total y tres de sus componentes (gastos administrativos, transferencias y gasto en obras públicas), así como sobre la deuda pública. La fuente de estos datos son las estadísticas de las "Finanzas públicas estatales y municipales" (Instituto Nacional de Estadística y Geografía, 2013) de diferentes años. ${ }^{17}$

Un primer aspecto importante que debe subrayarse es que las elecciones de gobernador en el Estado de México siempre han sido ganadas por los candidatos del PRI. De hecho, este partido nunca ha enfrentado una competencia real

\footnotetext{
${ }^{17}$ El periodo de análisis está determinado por la disponibilidad de información. Se ha tratado de tener un periodo de tiempo lo más largo posible para ganar grados de libertad en las estimaciones econométricas. El inconveniente es que sólo se ha podido construir series para un número limitado de componentes del gasto público estatal. Los cálculos se han hecho con base en datos a precios constantes de 2003 (Instituto Nacional de Estadística y Geografía, 2013).
} 
en los comicios hasta ahora celebrados. ${ }^{18}$ Los años de elección, los gobernadores y los periodos de gobierno se muestran en el cuadro 1 . Se puede apreciar que ha habido seis elecciones e igual número de periodos de gobierno, aunque ha habido nueve titulares de la gubernatura, debido a que tres de los electos renunciaron antes de concluir su gestión para asumir puestos en el gobierno federal.

Cuadro 1. Estado de México: Gobernadores, año de elección y periodo de gobierno, 1980-2011

\begin{tabular}{c|l}
\hline Año de elección & \multicolumn{1}{|c}{ Gobernador y periodo } \\
\hline 1981 & Alfredo del Mazo, 1981-1986 (Alfredo Baranda, 1986-1987) \\
1987 & Mario Ramón Beteta 1987-1989 (Ignacio Pichardo, 1989-1993) \\
1993 & Emilio Chuayffet, 1993-1995 (César Camacho, 1995-1999) \\
1999 & Arturo Montiel, 1999-2005 \\
2005 & Enrique Peña, 2005-2011 \\
2011 & Eruviel Ávila Villegas \\
\hline
\end{tabular}

Nota: Se señalan entre paréntesis los gobernadores sustitutos (no electos).

Fuente: Elaboración propia con base en González (2008).

Por su parte, las tasas de crecimiento promedio y la volatilidad del gasto y del PIB nacional y estatal se muestran en el cuadro 2. Destacan las elevadas tasas de crecimiento del gasto total en los gobiernos de Mario Ramón Beteta (relevado por Ignacio Pichardo) y Arturo Montiel, con $19.8 \%$ y $10.8 \%$ promedio anual, respectivamente. En un nivel desagregado, sobresalen las tasas de crecimiento de las transferencias por encima de las de los demás rubros. En particular, el crecimiento promedio del gasto en transferencias alcanzó casi $46 \%$ anual durante el periodo de Beteta (Pichardo), seguido por el de Emilio Chuayffet (sustituido por César Camacho), que fue mayor a 37\% anual. En el rubro de deuda pública, la tasa de crecimiento más alta (superior a $25 \%$ anual) se experimentó en el régimen de Arturo Montiel, en tanto que la mayor de obra pública (24.8\%) se presentó en el sexenio de Enrique Peña.

\footnotetext{
${ }^{18}$ Aunque autores como Padilla (2007) sostienen que las elecciones de gobernador han sido a veces muy competidas, los candidatos del PRI siempre han ganado por márgenes amplios (1981: 82.09\% de la votación estatal; 1987: 71.54\%; 1993: 62.36\%; 1999: 42.44\%, y 2005: 47.57\%) (Padilla, 2007; IEEM, 2016).
} 
Cuadro 2. Estado de México: Media y desviación estándar del gasto público y del PIB por sexenio, 1980-2011

\begin{tabular}{|c|c|c|c|c|c|c|c|c|c|c|c|c|}
\hline & \multicolumn{2}{|c|}{$1980-2011$} & \multicolumn{2}{|c|}{ 1981-1987 } & \multicolumn{2}{|c|}{ 1987-1993 } & \multicolumn{2}{|c|}{ 1993-1999 } & \multicolumn{2}{|c|}{ 1999-2005 } & \multicolumn{2}{|c|}{ 2005-2011 } \\
\hline & $\begin{array}{l}\frac{\pi}{0} \\
\frac{0}{\Sigma}\end{array}$ & 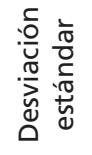 & $\begin{array}{l}\frac{\pi}{0} \\
\frac{0}{2}\end{array}$ & 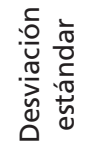 & $\begin{array}{l}\frac{\pi}{\bar{\theta}} \\
\frac{\mathbb{\theta}}{\Sigma}\end{array}$ & 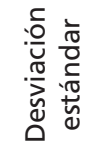 & $\begin{array}{l}\frac{\pi}{\pi} \\
\frac{0}{\alpha}\end{array}$ & 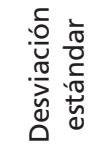 & $\frac{\frac{\pi}{0}}{\frac{0}{\Sigma}}$ & 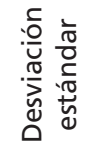 & $\begin{array}{l}\frac{\pi}{0} \\
\frac{0}{2}\end{array}$ & 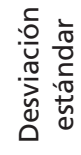 \\
\hline Gasto total & 6.50 & 15.01 & -8.21 & 14.08 & 19.75 & 18.67 & 5.61 & 8.45 & 10.75 & 8.72 & 7.07 & 8.98 \\
\hline $\begin{array}{l}\text { Gasto } \\
\text { administrativo }\end{array}$ & 8.13 & 23.13 & -0.17 & 17.37 & 19.07 & 6.36 & 13.18 & 49.59 & 7.30 & 6.08 & 2.66 & 2.13 \\
\hline Transferencias & 24.22 & 65.71 & 17.13 & 90.17 & 45.94 & 57.76 & 37.30 & 104.75 & 12.02 & 17.01 & 9.91 & 16.84 \\
\hline Obras públicas & 4.92 & 35.59 & -3.65 & 31.57 & 4.74 & 17.40 & -6.44 & 25.79 & 6.55 & 28.63 & 24.84 & 62.28 \\
\hline Deuda pública & 0.93 & 42.73 & 1.80 & 42.28 & -17.05 & 48.59 & -0.05 & 29.30 & 25.88 & 65.52 & -6.08 & 10.09 \\
\hline PIB nacional & 2.49 & 3.54 & 1.25 & 4.15 & 3.69 & 1.31 & 3.31 & 4.82 & 2.28 & 2.60 & 2.14 & 4.23 \\
\hline PIB estatal & 2.43 & 4.60 & 0.94 & 6.12 & 2.11 & 1.96 & 3.41 & 6.40 & 2.70 & 3.07 & 3.25 & 4.76 \\
\hline
\end{tabular}

Fuente: Estimaciones propias con datos de Germán (2005) e Instituto Nacional de Estadísticas y Geografía.

Por el contrario, las menores tasas de crecimiento se registraron durante los años ochenta, como consecuencia de los efectos de los programas de ajuste que se instrumentaron a nivel nacional para estabilizar la economía (Cárdenas, 1996). ${ }^{19}$ Específicamente, en el sexenio de 1981 a 1987, de Alfredo del Mazo (concluido por Alfredo Baranda), el gasto total disminuyó a una tasa media anual de $8.2 \%$, en tanto que los gastos administrativos y de obras públicas decrecieron a tasas promedio de $0.17 \%$ y $3.65 \%$, respectivamente. No obstante, llama la atención que el rubro de transferencias haya crecido a una tasa media anual de 17.1\%.

Por otra parte, es interesante hacer notar el manejo responsable de la deuda pública en la mayor parte del periodo estudiado. Durante los primeros dos sexenios, su crecimiento fue muy bajo y en los siguientes dos, incluso decreció. Fue hasta el gobierno de Montiel que la deuda alcanzó tasas elevadas (de 25.9\%

\footnotetext{
${ }^{19}$ De hecho, como se observa en el cuadro 2, las tasas de crecimiento más bajas del PIB nacional y estatal se presentaron durante 1981-1987, lapso que coincide con la peor fase de la crisis mexicana de los ochenta. Como se recordará, 1982 marca el inició de la crisis de la deuda y 1987 fue el año en que se instrumentó el Pacto de Solidaridad Económica, con el que se inició la estabilización macroeconómica (Cárdenas, 1996).
} 
Gráfica 1. PIB y gasto público total del Estado de México, 1980-2011

(tasas de crecimiento porcentual)

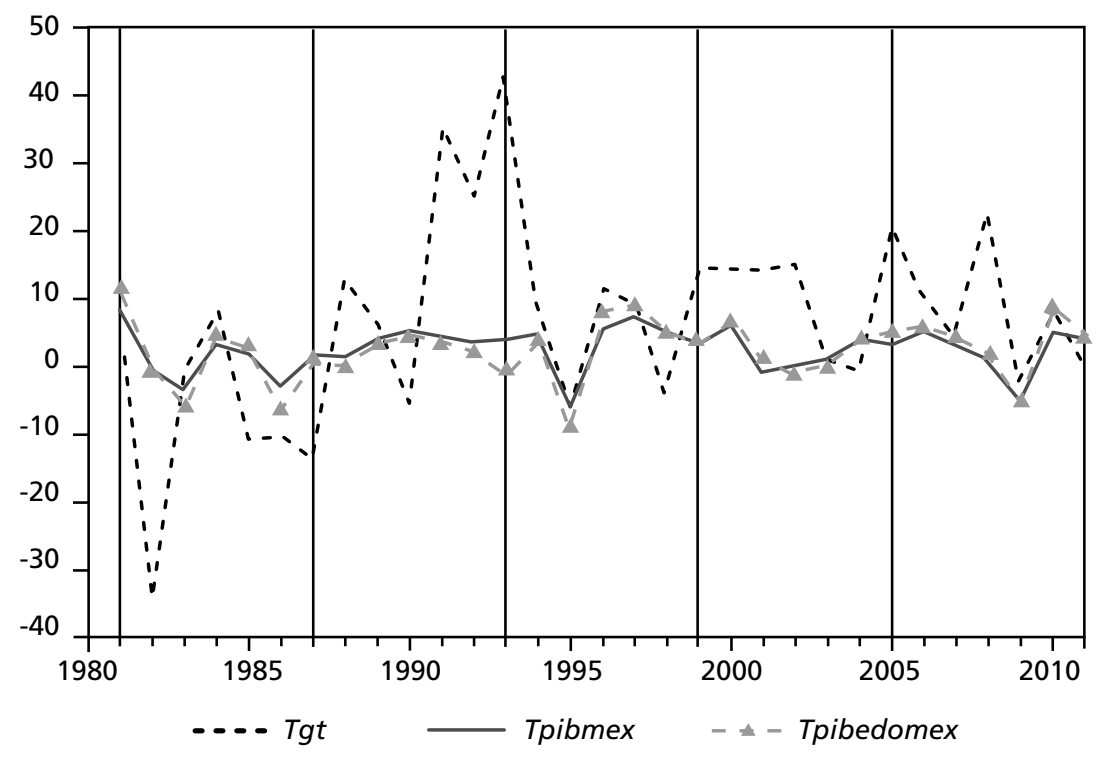

anual), lo que obligó a Peña a adoptar una política de reducción de deuda durante su periodo.

Estas significativas variaciones en las tasas de crecimiento generaron diferentes grados de volatilidad en las distintas variables. No obstante, el rubro de transferencias fue el más volátil en cuatro de los seis sexenios; solamente en los gobiernos de Montiel y Peña, los rubros de deuda y obra pública tuvieron desviaciones estándar mayores. Nótese que las desviaciones estándar de los componentes del gasto exceden con mucho a las del PIB nacional y estatal, lo que podría estar reflejando el alto grado de discrecionalidad en el manejo de los primeros.

El comportamiento de las tasas de crecimiento del gasto total y de sus componentes se presenta en las gráficas 1 y 2 , donde los años de elección se indican con líneas verticales; en la primera se presentan, también, las tasas de crecimiento del PIB nacional y estatal. Se observa que el comportamiento de las tasas estatales sigue muy de cerca al de las nacionales y que hay una relación muy baja entre el gasto total y el PIB en cualquiera de los dos ámbitos territoriales. Además, y de manera central para el tema analizado, se pueden ver tasas de crecimiento mayores en los años previos y en los de elección en varios rubros del gasto, así como tasas menores en los años que siguen a los comicios. Por ejemplo, el gasto en transferencias muestra tasas notablemente elevadas en los años de las eleccio- 
Gráfica 2. Componentes del gasto público del Estado de México, 1980-2011 (tasas de crecimiento porcentual)

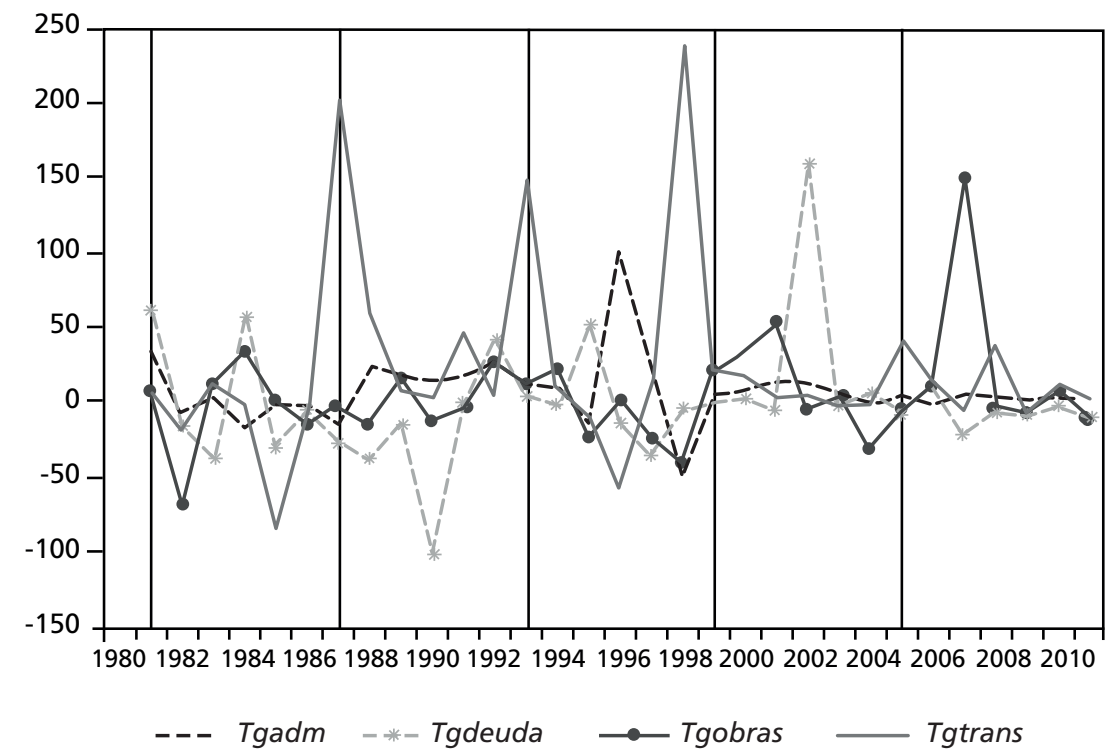

nes de 1987 y 1993, así como en las de 2005, aunque en menor medida; la tasa más alta se presenta en el año previo a las elecciones de 1999. Aunque en una proporción mucho menor, las tasas de crecimiento del gasto en obra púbica son mayores en el año de elección que en los años anteriores, pero también presentan un decrecimiento posterior en la mayoría de los casos.

Un análisis más detallado para identificar patrones consistentes con el CPP se presenta en los paneles de las gráficas 3 y 4 . En la primera de ellas, se muestra el comportamiento de cada variable en torno a las distintas elecciones consideradas (dos años antes y dos después), en tanto que en la segunda aparece el comportamiento del conjunto de variables analizadas para cada elección. Estas representaciones tratan de captar dicho comportamiento de acuerdo a la especificación que se ha hecho de las variables electorales en los modelos 1 y 2 , respectivamente.

En los paneles de la gráfica 3 se puede ver que, exceptuando la elección de 1987, el gasto público total tiene un comportamiento consistente con el CPP. Por su parte, el gasto en transferencias presenta tasas de crecimiento elevadas en los años previos y en los de elección, pero menores una vez celebrados los comicios (excluyendo el año de 1999, cuando la contracción se presenta antes de las 
Gráfica 3. Gasto público total y sus componentes antes, durante y después de cada elección en el Estado de México

(tasas de crecimiento porcentual)

a) Gasto total

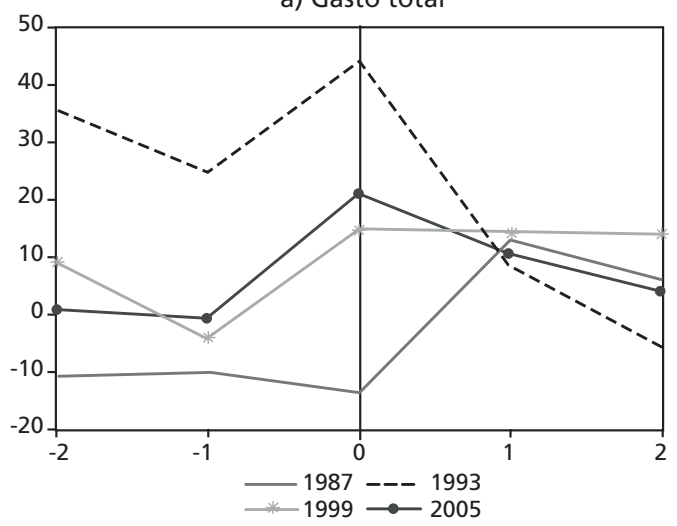

b) Gasto administrativo

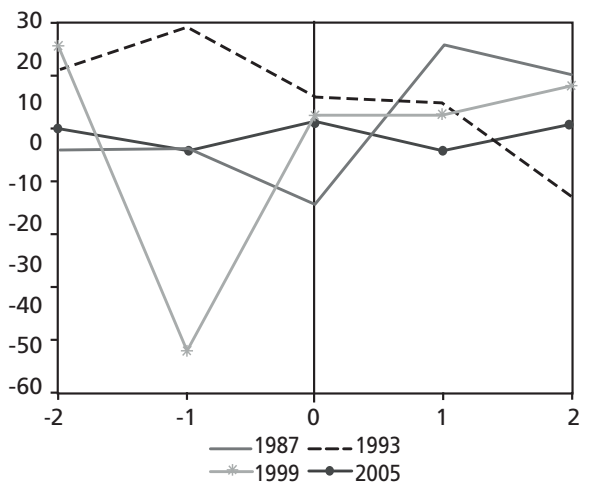

b) Gasto en obras públicas

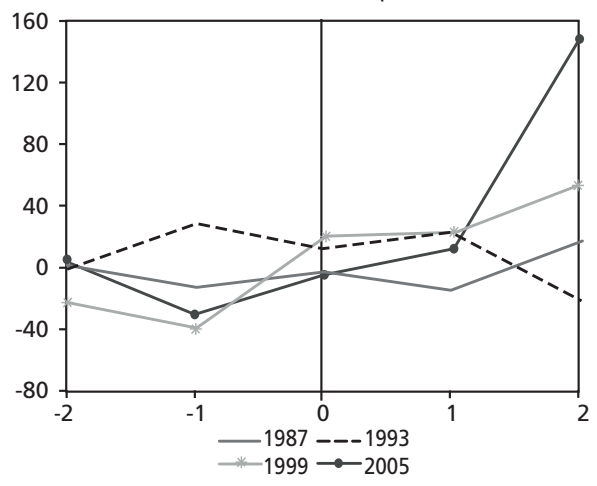

c) Gasto en transferencias y programas sociales

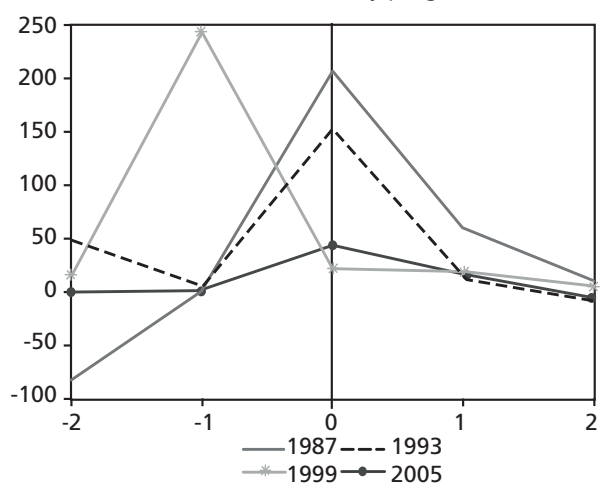

c) Deuda pública

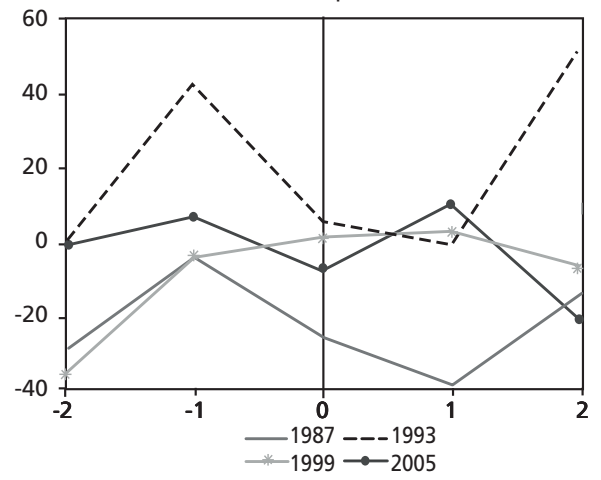


Gráfica 4. Gasto público total y sus componentes, por año electoral en el Estado de México

(tasas de crecimiento)
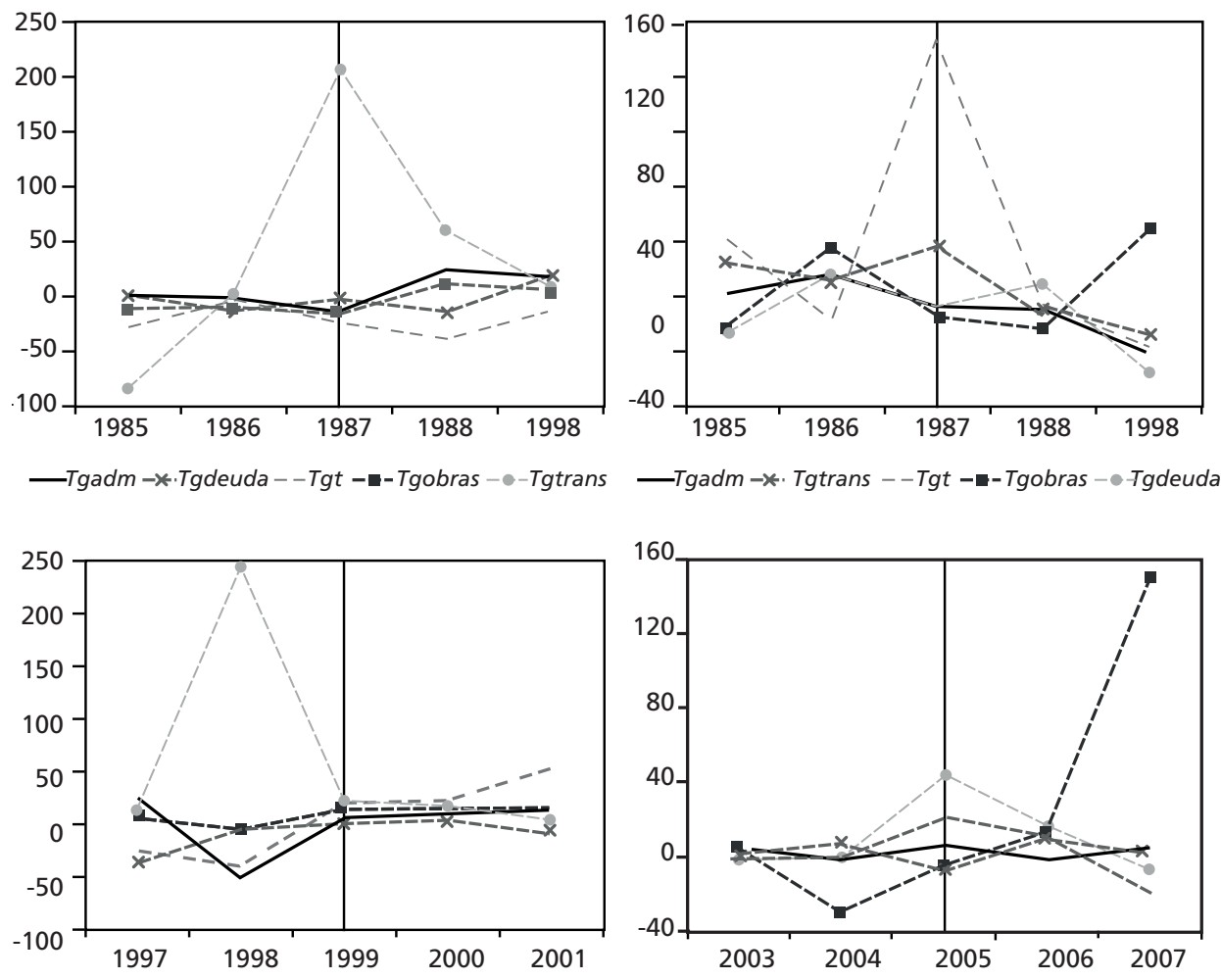

-Tgadm -*.Tgdeuda--Tgt -E-Tgobras--Tgtrans

-Tgadm $\rightarrow$-Tgdeuda $--T g t-\mathbf{E}-$ Tgobras - - Tgtrans

votaciones y no después). Asimismo, el gasto en obra pública muestra dicho comportamiento alrededor de las elecciones de 1987 y 1999. Durante estas últimas, el gasto administrativo tiene un comportamiento similar. Por su parte, en los paneles de la gráfica 4 destacan las tasas de crecimiento del gasto en trasferencias y, en menor proporción, las del gasto total, mientras que la deuda pública presenta un incremento constante alrededor de las elecciones de 2005.

El análisis previo sugiere la presencia del patrón previsto por la teoría del CPP en varios casos. No obstante, esta evidencia es simplemente episódica y, aunque interesante e intuitivamente atractiva, no puede considerarse como definitiva. A continuación, se presentan los resultados de la estimación econométrica para determinar si estos patrones de comportamiento han sido sistemáticos. 


\section{Análisis econométrico del CPP en el Estado de México}

Las estimaciones econométricas se muestran en los cuadros 3 y 4 . Como se mencionó antes, se partió de los modelos generales definidos en las ecuaciones (1) y (2), aunque los modelos finales se obtuvieron aplicando la metodología de lo general a lo particular (Charemza y Deadman, 1997). Se realizaron pruebas de autocorrelación, heteroscedasticidad, normalidad y especificación funcional; los valores $p$ correspondientes se presentan en el cuadro Al del anexo. En general, los modelos muestran una especificación aceptable, aunque persisten algunos problemas ${ }^{20}$ y a pesar de las dificultades en el modelaje asociadas a la existencia de tasas de crecimiento con valores anómalos en diversos momentos, lo cual se ha traducido en problemas de normalidad en los residuos. Para reducir tales valores, se introdujeron variables dicótomas adicionales, las cuales resultaron estar vinculadas a conductas oportunistas, como se discute más adelante. ${ }^{21}$ Por otro lado, cuando la prueba de White (1980) lo ha indicado, se han estimado los errores estándar consistentes en presencia de heteroscedasticidad aplicando la matriz de corrección sugerida por el mismo autor.

Las estimaciones obtenidas para los parámetros de los modelos revelan varios aspectos interesantes. En primer lugar, las series presentan escasa persistencia, pues sólo los gastos administrativos requieren de un término autorregresivo, el cual es significativo y menor a uno, lo que sugiere estacionariedad de ese componente del modelo. En segundo lugar, aunque estos resultados no son robustos, en el modelo 1, el crecimiento del PIB nacional afecta positivamente a la deuda, en tanto que, en el modelo 2, la misma variable afecta negativamente a los gastos administrativos y a los de transferencias. Por su parte, el crecimiento del PIB estatal presenta efectos positivos sobre el gasto total, un resultado que es consistente en las dos especificaciones. Efectos del mismo signo se presentan en los casos de gastos administrativos, transferencias y obra pública, aunque con diferentes horizontes temporales. En general, se podría considerar que estos re-

\footnotetext{
${ }^{20}$ El principal problema remanente, que ha sido difícil de erradicar, es el de la forma funcional, detectado con la prueba de Ramsey (1969). No obstante, como es bien sabido, esta prueba no identifica la causa específica del problema y, por ello, no da sugerencias sobre cómo resolverlo. Es difícil explicar las causas de los problemas de especificación persistentes, pero parece plausible atribuírselos a la discrecionalidad en el manejo del gasto público, en el que puede haber cambios drásticos de un año a otro, como se muestra en las características estadísticas básicas de las series, tal como Reyes y Mejía (2016) lo han sugerido.

${ }^{21}$ Las fechas de los valores anómalos en los residuos se muestran en el cuadro A2 del anexo.
} 
226 ECONOMÍA: TEORÍA Y PRÁCTICA • Nueva Época, número 45, julio-diciembre 2016

Cuadro 3. Resultados de la estimación del modelo 1 de CPP en el Estado de México

\begin{tabular}{|c|c|c|c|c|c|}
\hline & Total & $\begin{array}{c}\text { Gasto } \\
\text { administrativo }\end{array}$ & Transferencias & Obra pública & Deuda \\
\hline$\phi_{0}$ & $\begin{array}{c}0.944 \\
(0.7549) \\
\end{array}$ & $\begin{array}{c}6.962 \\
(0.017) \\
\end{array}$ & $\begin{array}{l}10.345 \\
(0.1767) \\
\end{array}$ & $\begin{array}{c}0.067 \\
(0.988) \\
\end{array}$ & $\begin{array}{r}-10.610 \\
(0.084) \\
\end{array}$ \\
\hline$\phi_{2}$ & & $\begin{array}{c}-0.296 \\
(0.011)\end{array}$ & & & \\
\hline$\alpha_{3}$ & & & & & $\begin{array}{c}2.049 \\
(0.061)\end{array}$ \\
\hline$\beta_{0}$ & $\begin{array}{c}1.374 \\
(0.034)\end{array}$ & & & & \\
\hline$\beta_{2}$ & $\begin{array}{c}1.117 \\
(0.068)\end{array}$ & & & & \\
\hline$\gamma_{a}$ & & & & & $\begin{array}{l}21.065 \\
(0.090)\end{array}$ \\
\hline$\theta_{1} 1993$ & $\begin{array}{c}33.420 \\
(0.006) \\
\end{array}$ & & & & \\
\hline$\theta_{2} 1996$ & & $\begin{array}{l}95.984 \\
(0.000) \\
\end{array}$ & & & \\
\hline$\theta_{3} 1987$ & & & $\begin{array}{r}197.626 \\
(0.000) \\
\end{array}$ & & \\
\hline$\theta_{4} 1998$ & & & $\begin{array}{r}232.647 \\
(0.000) \\
\end{array}$ & & \\
\hline$\theta_{5} 2007$ & & & & $\begin{array}{c}150.507 \\
(0.000) \\
\end{array}$ & \\
\hline$\theta_{6} 1990$ & & & & & $\begin{array}{r}-91.750 \\
(0.001)\end{array}$ \\
\hline$\theta_{7} 2002$ & & & & & $\begin{array}{r}162.177 \\
(0.000)\end{array}$ \\
\hline
\end{tabular}

Nota: Los coeficientes $\phi_{i}, \alpha_{i}, \beta_{i}$ y $\gamma_{i}$ corresponden a lo explicado sobre la ecuación (1). Los coeficientes $\theta_{i}$ corresponden a variables dicótomas que tratan de captar los efectos de los valores anómalos mostrados en el cuadro A.2 del anexo: la variable correspondiente toma el valor de 1 en el año indicado en la primera columna, junto a la variable respectiva, y 0 en cualquier otro caso. Los valores $p$ asociados aparecen entre paréntesis.

sultados constituyen evidencia que apoya la hipótesis de que el gasto público estatal es procíclico con respecto al PIB estatal, mientras que la deuda pública es procíclica, mientras que los gastos administrativos y las transferencias, son contracíclicos, en relación al PíB nacional. No obstante, es indudable que se requiere investigación adicional sobre este tema.

Los coeficientes de las variables electorales, a su vez, resultaron estadísticamente significativos en pocos casos. Específicamente, de acuerdo al modelo 1, 
Cuadro 4. Resultados de la estimación del modelo 2 del CPP en el Estado de México

\begin{tabular}{|c|c|c|c|c|c|}
\hline & Total & $\begin{array}{c}\text { Gasto } \\
\text { administrativo }\end{array}$ & Transferencias & Obra pública & Deuda \\
\hline$\phi_{0}$ & $\begin{array}{c}1.493 \\
(0.602) \\
\end{array}$ & $\begin{array}{c}3.492 \\
(0.380) \\
\end{array}$ & $\begin{array}{c}0.425 \\
(0.958) \\
\end{array}$ & $\begin{array}{c}-7.184 \\
(0.1106) \\
\end{array}$ & $\begin{array}{l}-1.048 \\
(0.832) \\
\end{array}$ \\
\hline$\phi_{2}$ & & $\begin{array}{l}-0.322 \\
(0.008)\end{array}$ & & & \\
\hline$\alpha_{3}$ & & & $\begin{array}{l}-12.223 \\
(0.005) \\
\end{array}$ & & \\
\hline$\beta_{0}$ & & $\begin{array}{l}-3.909 \\
(0.072) \\
\end{array}$ & & & \\
\hline$\beta_{2}$ & $\begin{array}{c}1.345 \\
(0.029) \\
\end{array}$ & & $\begin{array}{l}16.143 \\
(0.004)\end{array}$ & & \\
\hline$\beta_{3}$ & $\begin{array}{c}1.403 \\
(0.019) \\
\end{array}$ & & & & \\
\hline$\delta_{1}$ & & $\begin{array}{c}5.359 \\
(0.0664)\end{array}$ & & $\begin{array}{c}3.441 \\
(0.003)\end{array}$ & \\
\hline$\delta_{2}$ & $\begin{array}{l}-15.788 \\
(0.015) \\
\end{array}$ & $\begin{array}{l}-17.498 \\
(0.027) \\
\end{array}$ & & & \\
\hline$\theta_{1} 1993$ & $\begin{array}{l}15.774 \\
(0.013) \\
\end{array}$ & & & & \\
\hline$\theta_{2} 1996$ & & $\begin{array}{l}76.520 \\
(0.000)\end{array}$ & & & \\
\hline$\theta_{3} 1987$ & & & $\begin{array}{l}193.592 \\
(0.000) \\
\end{array}$ & & \\
\hline$\theta_{4} 1998$ & & & $\begin{array}{c}219.603 \\
(0.000)\end{array}$ & & \\
\hline$\theta_{5} 2007$ & & & & $\begin{array}{c}143.809 \\
(0.000) \\
\end{array}$ & \\
\hline$\theta_{6} 1990$ & & & & & $\begin{array}{l}-98.952 \\
(0.001)\end{array}$ \\
\hline$\theta_{7} 2002$ & & & & & $\begin{array}{l}259.197 \\
(0.000)\end{array}$ \\
\hline
\end{tabular}

Nota: Los coeficientes $\phi_{i}, \alpha_{i}, \beta_{i}$ y $\delta_{i}$ corresponden a lo explicado sobre la ecuación (2). Los coeficientes $\theta_{i}$ corresponden a variables dicótomas que tratan de captar los efectos de los valores anómalos mostrados en el cuadro A. 2 del anexo: la variable correspondiente toma el valor de 1 en el año indicado en la primera columna, junto a la variable respectiva, y 0 en cualquier otro caso. Los valores $p$ asociados aparecen entre paréntesis.

sólo ocurre un incremento significativo antes de las elecciones en la tasa de crecimiento de la deuda pública. Este resultado coincide con las conclusiones de Ramírez y Erquizio (2012), respecto al incremento de la deuda real como factor determinante del incremento del gasto estatal real. Resulta sorprendente que no se presente un patrón consistente con el CPP en los casos de los gastos en obras públicas y las transferencias, variables que por su visibilidad son ampliamente 
usadas con fines electorales. En cuanto a estos rubros, hay coincidencia con los resultados encontrados por Ramírez y Erquizio (2012) y Gámez e Ibarra-Yúnez (2009), quienes tampoco encuentran evidencia favorable al CPP.

Las estimaciones del modelo 2, por su parte, no son mejores. Aunque los coeficientes de las variables dicótomas vinculadas a las elecciones de 1987 son estadísticamente significativos en los casos del gasto total y de los gastos administrativos, su signo es contrario al sugerido por la teoría. Luego entonces, al parecer sólo existe evidencia de manipulación electoral del gasto total en la elección de Chuayffet, en 1993.

En principio, estos resultados constituyen evidencia débil en favor del CPP en el Estado de México. Sin embargo, un análisis detallado de los residuos de los modelos mostró la presencia de valores anómalos, lo que nos llevó a introducir variables dicótomas en los años y variables indicados en el cuadro A2 del anexo. ${ }^{22}$ Es importante subrayar que la variable dicótoma que vale 1 en 1993, año de la elección de Chuayffet, tiene un coeficiente estadísticamente significativo en el caso del gasto total. Más aún, las variables de 1987, año de elección de Beteta, y de 1998, año previo a la elección de Montiel, también tienen coeficientes significativos en los modelos de las transferencias. Como se puede ver en los cuadros 3 y 4 , los valores estimados de los coeficientes para esas observaciones anómalas son de una magnitud considerable, lo que no solamente distorsionaba las estimaciones y afectaba las propiedades de los modelos, sino que también dificultaba la identificación de un efecto sistemático de las elecciones sobre la dinámica del gasto y sus componentes. Más aún, las estimaciones asociadas a los efectos de esos valores anómalos se pueden vincular a la manipulación electoral del gasto total en una elección y del rubro de transferencias en al menos dos elecciones, lo que aporta evidencia adicional en favor del cPP en el Estado de México. ${ }^{23}$

Así pues, estos resultados sugieren que diversos componentes del gasto público se han manejado con objetivos electorales en diferentes episodios, pero

\footnotetext{
${ }^{22}$ De acuerdo con el hecho de que aproximadamente $99.9 \%$ de las observaciones de una distribución normal se ubican en un intervalo de tres desviaciones estándar en torno a la media, un valor anómalo se define como aquel que cae fuera de tal intervalo. Para captar su efecto, se introducen variables dicótomas que toman el valor de 1 en el año correspondiente al valor anómalo y 0 en cualquier otro caso. El valor del coeficiente estimado de estas variables mide su efecto en la tasa de crecimiento del rubro de gasto público correspondiente.

${ }^{23}$ En general, esta conclusión coincide con los resultados de Ramírez y Erquizio (2012), quienes muestran evidencia de CPP en el gasto en transferencias durante el año electoral en los 14 estados gobernados por el PRI.
} 
no reflejan patrones consistentes con la teoría del $\mathrm{CPP}^{24}$ Tampoco hay evidencia de que la mayor competencia que ha enfrentado el PRI en algunas elecciones -como las de 1987, 1993 y 2005-, la creación de órganos electorales autóno$\operatorname{mos}^{25}$ o la mayor educación o participación política hayan modificado las conductas oportunistas del partido en el poder, como lo muestra el hecho de que hay alguna evidencia de CPP en las diferentes elecciones a lo largo de todo el periodo de análisis: las variables electorales del modelo 2 podrían haber captado los efectos de tales cambios a través del tiempo en caso de haberse presentado.

\section{CONCLUSiones}

El objetivo de este trabajo ha sido verificar la existencia de ciclos político presupuestales en el gasto público total y tres de sus componentes, así como en la deuda pública del Estado de México, durante el período 1980-2011. Para ello, se analizaron las características de los datos en busca de evidencia preliminar y se encontró que las tasas de crecimiento de los rubros considerados muestran una alta volatilidad, sesgo positivo y valores anómalos. Estas propiedades se pueden asociar, por un lado, a un alto grado de discrecionalidad en el manejo del gasto y, por otro, a su uso con fines electorales, ya que los valores anómalos se pueden vincular a elevadas tasas de crecimiento en años de comicios o en años previos. En particular, el gasto total presenta tasas mayores a la media en torno a las elecciones de 1993 (especialmente), 1999 y 2005, en tanto que el rubro de transferencias presenta tasas altísimas en relación con las elecciones de 1987, 1993 y 1999. La evidencia en el caso de otros rubros y de otras elecciones es menos concluyente.

Para verificar de manera formal estos hallazgos, se estimaron dos tipos de modelos autorregresivos extendidos. En el primero, se utilizan variables dicótomas que tratan de distinguir el comportamiento de las tasas de crecimiento del

\footnotetext{
${ }^{24}$ Un factor adicional que puede explicar la ausencia de CPP en los resultados es el uso de información anual que impide distinguir claramente la dinámica del gasto un año antes y un año después de las elecciones, problema que se conoce como de agregación temporal (Streb, Lema y Garofalo, 2012).

${ }^{25}$ En 1996 desapareció la Comisión Estatal Electoral -en la que intervenía el gobierno localpara dejar su lugar a un organismo público especializado, denominado Instituto Electoral del Estado de México (IEEM), reconociéndolo como órgano responsable de la organización, desarrollo y vigilancia de los procesos electorales en la entidad (IEEM, 2015). Véase Padilla (2007) para un análisis detallado de las condiciones institucionales y el grado de competencia en las elecciones de gobernador recientes en el Estado de México.
} 
gasto antes, durante y después de las elecciones, tal como se hace en la mayoría de los trabajos publicados sobre el tema. Con este modelo, solamente se identificó un incremento significativo en la tasa de crecimiento de la deuda pública antes de las elecciones. En el segundo modelo, se utilizaron variables binarias que describen el patrón del CPP para cada elección; en este caso, se encontró evidencia de manipulación electoral del gasto total en la elección de 1993. En general, ha sido difícil identificar efectos sistemáticos y significativos de las elecciones en las tasas de crecimiento de los rubros de gasto analizados, debido a sus propiedades mencionadas más arriba. Sin embargo, precisamente ese comportamiento no estándar ofreció la posibilidad de encontrar evidencia adicional sobre el CPP. En particular, las tasas de crecimiento extraordinariamente elevadas de diversos rubros del gasto (valores anómalos) se modelaron como variables dicótomas, las cuales no solamente resultaron con coeficientes estadísticamente significativos, sino que se pudieron vincular al manejo electoral del gasto total en la elección de 1993 y de las transferencias en las elecciones de 1987 y 1999.

Estos resultados, sin embargo, no pueden considerarse como evidencia definitiva del manejo oportunista del gasto público durante las elecciones mexiquenses de gobernador, aun cuando exista una percepción generalizada de que así es. En general, el cPP es un fenómeno difícil de aprehender, debido a múltiples factores, entre los que se cuenta la contabilización de gastos electorales en otros rubros del presupuesto o su diferimiento a otros ejercicios fiscales, además del ingreso de recursos financieros de fuentes no públicas difíciles de registrar. Es probable que varias, o todas, de estas maniobras hayan sido utilizadas en las elecciones de gobernador del Estado de México, una entidad de importancia estratégica en el plano nacional, cuyas administraciones han sido dominadas plenamente por el PRI. Como se ha mencionado previamente, la elección de gobernador en esa entidad se realiza un año antes que la del presidente de la República, por lo que al deseo de conservar la gubernatura estatal para el partido en el poder y a la necesidad de legitimar ante el electorado su permanencia, se suma la presión a nivel nacional para no perder la elección correspondiente. Todo ello, sin duda, podría traducirse en conductas oportunistas de los gobernantes. Sin embargo, la evidencia presentada en este documento no ofrece un sustento definitivo a esta conjetura, por lo que resulta necesario seguir investigando sobre un tema tan evasivo como éste.

Lo que resulta evidente, y debe subrayarse, es que el uso del gasto público con fines electorales constituye un desperdicio social, que beneficia a grupos o partidos políticos específicos y resta eficacia a las políticas públicas estatales. 
Por ello, es imperativo continuar el análisis del CPP para comprobar su existencia de manera fehaciente y, con ello, definir controles efectivos que contrarresten su instrumentación y, eventualmente, permitan erradicarlo. Existen diferentes áreas de análisis que podrían explorarse, no sólo en el caso del Estado de México, sino para el resto del país. Algunas de ellas se relacionan con el estudio más detallado de elecciones particulares, especialmente las más recientes, para verificar si la creación de órganos electorales autónomos ha contribuido al menor uso oportunista de los recursos públicos o si el más alto nivel educativo del electorado y la disponibilidad de información en cantidades superiores y de mejor calidad han significado una mayor competencia política para el partido en el poder y una fiscalización más efectiva. Adicionalmente, se podría indagar sobre la posibilidad de que el CPP se presente en rubros de política social. Éstas son solamente algunos temas de una agenda de investigación que seguramente crecerá de manera importante en el futuro inmediato, en la medida que aumenten las demandas por una democracia efectiva.

\section{Anexo}

Cuadro A1. Pruebas de especificación de los modelos (valores $p$ )

\begin{tabular}{|c|c|c|c|c|c|c|c|c|c|c|}
\hline \multirow{2}{*}{ Prueba } & \multicolumn{5}{|c|}{ Modelo 1} & \multicolumn{5}{|c|}{ Modelo 2} \\
\hline & Tgt & Tgadm & Tgtrans & Tgobras & Tgdeuda & Tgt & Tgadm & Tgtrans & Tgobras & Tgdeuda \\
\hline LM (1) & 0.633 & 0.682 & 0.905 & 0.952 & 0.843 & 0.1038 & 0.7734 & 0.9178 & 0.7258 & 0.4207 \\
\hline LM (2) & 0.439 & 0.164 & 0.964 & 0.728 & 0.839 & 0.2664 & 0.5338 & 0.9767 & 0.9310 & 0.4647 \\
\hline Ramsey (1) & 0.683 & 0.003 & n.a. & n.a. & 0.298 & 0.4008 & 0.0085 & 0.0005 & 0.4875 & n.a. \\
\hline Ramsey (2) & 0.648 & 0.002 & n.a. & n.a. & 0.582 & 0.5389 & 0.0000 & 0.0016 & 0.3490 & n.a. \\
\hline \multicolumn{11}{|l|}{ White } \\
\hline Con & 0.693 & 0.052 & 0.877 & 0.568 & 0.902 & 0.8808 & 0.0563 & 0.0762 & 0.9316 & 0.7100 \\
\hline $\operatorname{Sin}$ & 0.450 & 0.879 & 0.877 & 0.568 & 0.869 & 0.8019 & 0.0494 & 0.6661 & 0.8023 & 0.7100 \\
\hline ARCH (1) & 0.306 & 0.876 & 0.525 & 0.481 & 0.460 & 0.8450 & 0.9871 & 0.4216 & 0.7555 & 0.8678 \\
\hline $\mathrm{ARCH}(2)$ & 0.235 & 0.879 & 0.770 & 0.622 & 0.746 & 0.9358 & 0.8977 & 0.3679 & 0.9202 & 0.8714 \\
\hline Normalidad & 0.868 & 0.708 & 0.000 & 0.251 & 0.019 & 0.728950 & 0.330211 & 0.448796 & 0.464418 & 0.014 \\
\hline
\end{tabular}

Nota: Cuando la prueba de White lo indicó necesario, se estimaron los errores estándar consistentes en presencia de heteroscedasticidad. La prueba de Ramsey incluye 1 y 2 términos. Las estimaciones se hicieron en EViews 6.0. 
232 ECONOMÍA: TEORÍA Y PRÁCTICA • Nueva Época, número 45, julio-diciembre 2016

Cuadro A2.Valores anómalos de los residuos de los modelos 1 y 2

\begin{tabular}{c|cc}
\hline Variables & \multicolumn{2}{|c}{ Años } \\
\hline Tgt & 1993 & \\
Tgadm & 1996 & 1998 \\
Tgtrans & 1987 & \\
Tgobras & 2007 & 2002 \\
\hline Tgdeuda & 1990 & \\
\hline
\end{tabular}

\section{REFERENCIAS BIBLIOGRÁFICAS}

Alesina, Alberto (1987), "Macroeconomic Policy in a Two-party System as a Repeated Game”, Quarterly journal of Economics, 102 (3), pp 651-678.

(1989), "Politics and Business Cycles in Industrial Democracies", Economic Policy, 4 (8), pp. 55-98.

Alesina, Alberto, y Sachs, Jeffrey (1988), "Political Parties and the Business Cycle in the United States, 1948-1984”, Journal of Money Credit and Banking, 20 (1), pp. 63-82.

Argandoña, Antonio; Gámez, Consuelo, y Mochon, Francisco (1996), Macroeconomía avanzada, modelos dinámicos y teoría de la política económica, Madrid, Mc Graw Hill.

Assael, Paola, y Larraín, Felipe (1994), “El ciclo político económico: teoría, evidencia y extensión para una economía abierta”, Cuadernos de Economía, 31 (92), pp. 87-114.

Cárdenas, Enrique (1996), La política económica de México, 1950-1994, México, Fondo de Cultura Económica.

Charemza, Wojciech, y Deadman, Derek, F. (1997), New Directions in Econometric Practice: General to Specific Modelling, Cointegration and Vector Autoregression, Cheltenham, Edward Elgar.

Drazen, Allan (2001), “The Political Business Cycle after 25 Years”, en Ben S. Bernanke and Kenneth Rogoff (eds.), NBER Macroeconomics Annual 2000, Cambridge, The MIT Press, pp. 75-138.

Fontaine, Juan Andrés (1983), "El rol macroeconómico del Estado", Estudios Públicos, 0 (9), pp.1-23.

Gámez, Cesáreo (2010), El ciclo político y la economía mexicana, Monterrey, Universidad Autónoma de Nuevo León.

(2012), Política y economía. El ciclo sexenal en México, México, Plaza y Valdés. 
Gámez, Cesáreo, e Ibarra-Yúnez, Alejandro (2009), "El ciclo político oportunista y el gasto de los estados mexicanos", Gestión y Política Pública, 18 (1), pp. 39-65.

Germán, Vicente (2005), “Generación del producto interno bruto mexicano por entidad federativa, 1940-1992”, El Trimestre Económico, 72 (3), pp. 617-653.

González, María de los Angeles (2002), "Do Changes in Democracy Affect the Political Budget Cycle? Evidence from México", Review of Development Economics, 6 (2), pp. 204-224.

González, Sergio A. (2008), "Monografía política en el Estado de México (1981-2008)", documento de trabajo, El Colegio de México.

Heath, Jonathan (2000), La maldición de las crisis sexenales, Grupo Editorial Iberoamérica, México.

Hibbs, Douglas (1977), "Political Parties and Macroeconomic Policy", American Political Science Review, 71 (4), pp. 1467-1487.

IEEM (2015), "Historia”, en IEEM, Consultado el 28 de diciembre de 2015, en: http://www. ieem.org.mx/acerca/historia.html.

(2016), "Resultados electorales", en IEEM. Consultado el 5 de enero de 2016, en: http://www.ieem.org.mx/numeralia/result_elect.html.

Instituto Federal Electoral (2013), "Estadísticas y resultados electorales". Consultado el 15 de enero de 2013, en: http://www2.ife.org.mx/portal/site/ifev2/Estados/.

Instituto Nacional de Estadística y Geografía (2013), "Finanzas públicas estatales y municipales", en Banco de Información Económica. Consultado el 9 de enero de 2015, en: http://www.inegi.org.mx/sistemas/bie.

Larraín, Felipe, y Assael, Paola (1997), "El ciclo político económico en Chile desde 1939”, Estudios Públicos, 0 (68), pp. 197-214.

Lohmann, Susanne (1998), "Rationalizing the Political Business Cycle: a Workhorse Model", Economics and Politics, 0 (10), pp. 1-17.

Lustig, Nora (2002), México hacia la reconstrucción de una economía, México, Fondo de Cultura Económica/El Colegio de México.

Magaloni, Beatriz (2000), "Institutions, Political Opportunism and Macroeconomic Cycles: México 1970-1998", ponencia al XxII International Congress of the Latin American Studies Association, Miami, 16 al 18 de marzo de 2000.

McCallum, Bennett (1978), “The Political Bussiness Cycle: an Empirical Test”, Southern Economic Journal, 44 (3), pp 504-515.

Moreno-Brid, Juan Carlos, y Ros, Jaime (2010), Desarrollo y crecimiento en la economía mexicana, México, Fondo de Cultura Económica.

Muñoz, Rafael E. (2006), "Ciclos político-económicos: teoría y evidencia empírica”, Temas de Coyuntura, 0 (54), pp. 29-72. 
Nordhaus, William D. (1975), “The Political Business Cycle”, Review of Economic Studies, 0 (42), pp. 169-190.

Ogura, Laudo M. (2000), "Political Business Cycles in the Brazilian Economy (19801999)", manuscrito, Department of Economics, University of Brazilia.

Padilla, David (2007), "Procesos electorales en el Estado de México, 1981-2006”, Espacios Públicos, 10 (20), pp. 58-89

Ramírez, Roberto, y Erquizio, Alfredo (2012), “Análisis del ciclo político electoral a partir de variables de gasto público por entidad federativa en México, 1993-2009”, Paradigma Económico, 4 (2), pp. 5-27.

Ramsey, James B. (1969), “Tests for Specification Errors in Classical Linear Least Squares Regression Analysis", Journal of the Royal Statistical Society, 31 (2), pp. 350-371.

Remmer, Karen (1991), "The Political Impact of Economic Crisis in Latin America in the 1980s", American Political Science Review, 85 (3), pp. 777-800.

Reyes, Marlen R; Mejía, Pablo, y Riguzzi, Paolo (2013), “Ciclo político presupuestal y gobiernos con y sin mayoría en México, 1994 y 2006”, Economía Sociedad y Territorio, 13 (41), pp. 79-119.

Reyes, Marlen R, y Mejía, Pablo (2016), “Ciclo político presupuestal en México, 19802013: un enfoque ecométrico", Gestión y Política Pública, por publicarse.

Rogoff, Kenneth (1990), "Equilibrium Political Budget Cycles", The Review of Economic Studies, 89 (1), pp. 21-36

Rogoff, Kenneth, y Sibert, Anne (1988), "Elections and Macroeconomic Policy Cycles", The Review of Economic Studies, 55, pp. 1-16.

Schuknecht, Ludger (2000), "Fiscal Policy Cycles and Public Expenditure in Developing Countries", Public Choice, 0 (102), pp. 115-130.

Shi, Min, y Svensson, Jakob (2002), “Conditional Political Budget Cycles”, CEPR Discussion Paper 3352.

Streb, Jorge M.; Lema, Daniel, y Garofalo, Pablo (2012) “Temporal Aggregation in Political Budget Cycles", Economia, Journal of the Latin American and Caribbean Economic Association, 13, pp. 39-69.

Tufte, Edward R. (1978), Political Control of the Economy, Princenton, Princeton University Press.

Weatherford, Stephen M. (1987), "The Interplay of Ideology and Advine in Economic Policy-Making: the Case of Political Business Cycles", The Journal of Politics, 49, pp. 925-952.

White, Halber (1980), “A Heteroskedasticity-consistent Covariance Matrix and a Direct Test for Heteroskedasticity", Econometrica, 0 (8), pp. 817-838. 